\title{
$F$-region radio and optical measurement of nighttime TID campaign
}

\author{
Akinori Saito* \\ School of Electrical and Computer Engineering, Cornell University, Ithaca, NY 14853-3801, U.S.A.
}

1. Observations of traveling ionospheric disturbances and 3-m scale irregularities in the nighttime $F$-region ionosphere with the MU radar and a GPS network $\ldots \ldots \ldots \ldots \ldots \ldots \ldots \ldots \ldots \ldots \ldots \ldots \ldots \ldots \ldots \ldots \ldots \ldots$

2. Observations and modeling of $630 \mathrm{~nm}$ airglow and total electron content associated with

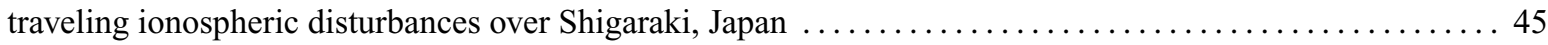

3. Imaging observations of the equatorward limit of midlatitude traveling ionospheric disturbances $\ldots \ldots \ldots 57$

4. A new technique for mapping of total electron content using GPS network in Japan $\ldots \ldots \ldots \ldots \ldots \ldots$

5. Plasmaspheric electron content in the GPS ray paths over Japan under magnetically quiet

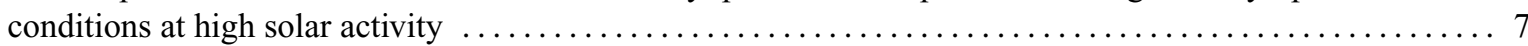

\section{Preface}

$F$-region Radio and Optical measurement of Nighttime TID (FRONT) campaign was conducted to clarify the physical mechanism of the Medium-Scale Traveling Ionospheric Disturbances (MSTIDs) with novel observational techniques. MSTIDs are migrating structures in the $F$-region ionosphere with about $300 \mathrm{~km}$ of wavelength and $100 \mathrm{~m} / \mathrm{s}$ of propagating velocity (Francis, 1974). Array of all-sky CCD cameras and GPS receivers observed two-dimensional structures of MSTIDs with wide field-of-view and high temporal and spatial resolutions in May 1998 and August 1999. Twodimensional observation made it possible to distinguish between the spatial structures and temporal evolutions of the ionospheric phenomenon.

In the last decade, several new features of the midlatitude ionosphere have been discovered by observations of radars, satellites, all-sky cameras and GPS receivers (e.g., Fukao et al., 1991; Ogawa et al., 1994; Miller et al., 1997; Garcia et al., 2000). Some of these new findings were stimulated by the discovery of the $F$-region field-aligned irregularities in the nighttime midlatitude ionosphere by the MU radar (Fukao et al., 1991). Their large Doppler velocity indicates that the intense polarization electric field is generated in the midlatitude ionosphere. The incoherent scatter observation of the Arecibo radar also detected the intense polarization electric field associating with MSTIDs in the nighttime midlatitude ionosphere (Miller et al., 1997). The generation and effect of the polarization field are not taken into account in the classical theory of the traveling ionospheric disturbances proposed by Hines (1974).

The recent instrumental advances have enabled all-sky CCD cameras to detect the two-dimensional structures of airglow with high spatial resolution. Dynamic behaviors

* On leave from Department of Geophysics, Graduate School of Science, Kyoto University, Kyoto 606-8502, JAPAN.

Copy right (c) The Society of Geomagnetism and Earth, Planetary and Space Sciences (SGEPSS); The Seismological Society of Japan; The Volcanological Society of Japan; The Geodetic Society of Japan; The Japanese Society for Planetary Sciences. of the meso-scale ionospheric structures in the nighttime midlatitude ionosphere were detected with an all-sky CCD camera at Arecibo (Kelley et al., 2000). The complex features of the meso-scale structures, such as MSTIDs, indicate the existence of an unknown interaction between the neutral atmosphere and the ionized atmosphere in the midlatitude ionosphere. To detect the two-dimensional structures of the ionosphere, GPS receiver array is another powerful tool that have been developed recently. GPS Earth Observation Network (GEONET) in Japan consists of more than one thousand of GPS receivers and has $1 \times 10^{6} \mathrm{~km}^{2}$ of field-of-view. Using the data of GEONET, the two-dimensional structures and time evolutions of Total Electron Content (TEC) disturbances were studied by Saito et al. (1998).

With these two novel observational techniques, FRONT campaign was carried out to study the two-dimensional structures of nighttime MSTIDs over Japan. To detect the other physical parameters of the ionosphere, the MU radar, ionosonde network and Fabry-Perot Interferometers (FPI) were operated in this campaign. The detail of the observational setup was summarized by Saito et al. (2001) and Saito et al. (2002). The preliminary results were reported by Saito et al. (2001), Kubota et al. (2000) and Shiokawa et al. (2000). The structures of the $630 \mathrm{~nm}$ band airglow were found to coincide with the structures of TEC and propagate to the southwest in about $100 \mathrm{~m} / \mathrm{s}$ of velocity. In these preliminary studies, the absolute value of TEC was not derived but only the perturbation component of TEC was studied because the observed TEC data contains ambiguities caused by the internal biases of the GPS receivers and transmitters. Otsuka et al. (2002) estimated these internal biases using an efficient procedure for GPS networks of significant number receivers, and evaluated its accuracy and errors using a simulation with the IRI ionospheric model. Another source of the error in the GPS-TEC data is the contribution of the plasmasphere. Using an ionosphere and plasmasphere model, the contribution of the plasmaspheric electron to the GPSTEC data was discussed by Balan et al. (2002).

In FRONT campaign, five and six all-sky CCD cameras 
were deployed for FRONT-1 (May 1998) and FRONT-2 (August 1999) campaign, respectively. The array of all-sky cameras observed the $630 \mathrm{~nm}$ band airglow with wide fieldof-view over Japan. Some portions of the field-of-views of cameras were overlapped each other. The altitude of the emission layer was determined by triangulation technique (Kubota et al., 2000). In FRONT-2 campaign, the behavior of MSTIDs around the boundary between the midlatitude and equatorial regions were focused to study the termination of MSTIDs. Shiokawa et al. (2002) clarified the airglow structures at the equatorial boundary of the midlatitude region using an all-sky CCD camera at Okinawa. The simultaneous observation of $630 \mathrm{~nm}$ band airglow and TEC made it possible to compare the absolute value of TEC and 630 $\mathrm{nm}$ airglow. Ogawa et al. (2002) evaluated the variations of TEC and airglow associated with MSTIDs using the neutral atmosphere model. The relation between the 3-m scale fieldaligned irregularities and MSTIDs were studied by Saito et al. (2002) using the data of the coherent scatter observation of the MU radar. The large-scale background ionospheric conditions of the occurrence of MSTIDs were also studied with the incoherent scatter observation of the MU radar.

The coordinated observation of GPS network, all-sky camera network, a coherent and incoherent radar, FPIs and ionosondes in FRONT campaign, and the comparison and evaluation of these observational results with atmospheric models clarified several new features of the nighttime midlatitude traveling ionospheric disturbances. The phenomenon was revealed to have wide spectrum from 3-m to 1000 $\mathrm{km}$ scale, and be generated by a non-linear interaction of the neutral atmosphere and the ionized atmosphere. Based on these observational results, it is necessary to develop a new theoretical framework to understand the ionospheric dynamics at midlatitudes.

\section{References}

Balan, N., Y. Otsuka, T. Tsugawa, S. Miyazaki, T. Ogawa, and K. Shiokawa, Plasmaspheric electron content in the GPS ray paths over Japan under magnetically quiet conditions at high solar activity, Earth Planets Space, 54, this issue, 71-79, 2002.

Francis, S. H., A theory of medium-scale traveling ionospheric disturbances, J. Geophys. Res., 79, 5245-5260, 1974.

Fukao, S., M. C. Kelley, T. Shirakawa, T. Takami, M. Yamamoto, T. Tsuda, and S. Kato, Turbulent upwelling of the mid-latitude ionosphere 1. ob- servational results by the MU radar, J. Geophys. Res., 96, 3725-3746, 1991.

Garcia, F. J., M. C. Kelley, J. J. Makela, and C.-S. Huang, Airglow observations of mesoscale low-velocity traveling ionospheric disturbances at mid-latitudes, J. Geophys. Res., 105, 18,407-18,416, 2000.

Hines, C. O., The upper atmosphere in motion, American Geophysical Union, Washington D. C., 1974.

Kelley, M. C., F. Garcia, J. Makela, T. Fan, E. Mak, C. Sia, D. Alcocer, Highly structured tropical airglow and TEC signatures during strong geomagnetic activity, Geophys. Res. Lett., 27, 465-468, 2000.

Kubota, M., K. Shiokawa, M. K. Ejiri, Y. Otsuka, T. Ogawa, T. Sakanoi, H. Fukunishi, M. Yamamoto, S. Fukao, A. Saito, Traveling ionospheric disturbances observed in the OI 630-nm nightglow images over Japan by using a multi-point imager network during the FRONT campaign, Geophys. Res. Lett., 27, 4037-4040, 2000.

Miller, C. A., W. E. Swartz, M. C. Kelley, M. Mendillo, D. Nottingham, J. Scali, and B. Reinisch, Electrodynamics of midlatitude spread $F$ 1. Observations of unstable, gravity wave-induced ionospheric electric fields at tropical latitudes, J. Geophys. Res., 102, 11,521-11,532, 1997.

Ogawa, T., K. Ohtaka, T. Takami, Y. Yamamoto, M. Yamamoto, and S. Fukao, Medium- and large-scale TIDs simultaneously observed by NNSS satellites and the MU radar, Low-Latitude Ionospheric Physics, edited by F. S. Kuo, Elsevier Science, Oxford, 1994.

Ogawa, T., N. Balan, Y. Otsuka, K. Shiokawa, C. Ihara, T. Shimomai, and A. Saito, Observations and modeling of $630 \mathrm{~nm}$ airglow and total electron content associated with traveling ionospheric disturbances over Shigaraki, Japan, Earth Planets Space, 54, this issue, 45-56, 2002.

Otsuka, Y., T. Ogawa, A. Saito, T. Tsugawa, S. Fukao, and S. Miyazaki, A new technique for mapping of total electron content using GPS network in Japan, Earth Planets Space, 54, this issue, 63-70, 2002.

Saito, A., S. Fukao, and S. Miyazaki, High resolution mapping of TEC perturbations with the GSI GPS network over Japan, Geophys. Res. Lett., 25, 3079-3082, 1998.

Saito, A., M. Nishimura, M. Yamamoto, M. Kubota, K. Shiokawa, Y. Otsuka, T. Tsugawa, S. Fukao, T. Ogawa, M. Ishii, T. Sakanoi, and S. Miyazaki, Traveling ionospheric disturbances detected in the FRONT campaign, Geophys. Res. Lett., 28, 689-692, 2001.

Saito, A., M. Nishimura, M. Yamamoto, S. Fukao, T. Tsugawa, Y. Otsuka, S. Miyazaki, and M. C. Kelley, Observations of traveling ionospheric disturbances and 3-m scale irregularities in the nighttime $F$-region ionosphere with the MU radar and a GPS network, Earth Planets Space, 54, this issue, 31-44, 2002.

Shiokawa, K., M. K. Ejiri, Y. Otsuka, T. Ogawa, M. Kubota, K. Igarashi, A. Saito, T. Nakamura, Multi-point observation of short-period mesospheric gravity waves over Japan during the FRONT campaign, Geophys. Res. Lett., 27, 4057-4060, 2000.

Shiokawa, K., Y. Otsuka, M. K. Ejiri, Y. Sahai, T. Kadota, C. Ihara, T. Ogawa, K. Igarashi, S. Miyazaki, and A. Saito, Imaging observations of the equatorward limit of midlatitude traveling ionospheric disturbances, Earth Planets Space, 54, this issue, 57-62, 2002.

A. Saito (e-mail: saito@ece.cornell.edu) 